\title{
Challenging Definitions of Psychological Trauma: Connecting Racial Microaggressions and Traumatic Stress
}

\author{
Kevin L. Nadal \\ Tanya Erazo \\ Rukiya King
}

John Jay College of Criminal Justice - City University of New York

\begin{abstract}
While previous studies have found significant relationships between racial microagressions, depression, and anxiety, few studies have examined the effects of racial microaggressions on traumatic stress. Furthermore, although trauma has been traditionally conceptualized as psychophysiological reactions to life-threatening events, the notion of racial trauma has been excluded, despite resulting in similar symptomatology. The current study utilized a correlational, cross-sectional design with a racially diverse sample of people of color $(N=254)$ to investigate the relationships between racial microaggressions, racially- or culturally-related trauma, and trauma symptoms. Using hierarchical multiple regression analysis, results indicated that a greater frequency of racial microaggressions was significantly associated with greater traumatic stress symptoms, and that school or workplace microaggressions were the type of microaggression that was most associated with traumatic symptoms. Implications are discussed, including the need for counselors, psychologists, and helping professionals to consider racial microaggressions as traumatic events while using culturally-informed traumafocused methods to normalize and empower people of color.
\end{abstract}

Keywords: microaggressions; discrimination; racism; trauma; racial trauma

(C) 2019 Nadal, Erazo, \& King.. Free to copy and share for education and scholarship under a Creative Commons Attribution NonCommercialNoDerivatives 4.0 License. 
Though slavery and segregation are no longer legal in the United States (US), there are many ways that racism manifests in our contemporary American society. First, while blatant racism had previously been suggested to have dwindled in the past (Sue et al., 2007), overt and intentional interpersonal racial discrimination remains prevalent in the US. For instance, the Southern Poverty Law Center reported a significant increase in race-based, ethnic-based, and religious-based hate crimes after the 2016 Presidential Election (Nadal, 2017). Second, some authors have described how racism is systemic and structural- manifesting through unjust institutional policies and societal norms. Examples include the lack of accountability for police violence or police misconduct towards Black people, the disproportionate incarceration of Black Americans in the criminal justice system, and the continuance of unequal health disparities for communities of color (Hargon et al., 2017; Jee-Lyn García \& Sharif, 2015). Systemic racism has also been used to explain economic and educational differentials between people of color and White Americans. The Bureau of Labor Statistics (2019) reported median weekly earnings for Black men were $74.7 \%$ of the median for White men, and median weekly earnings for Latino men were $\$ 728$, or $70.5 \%$ of the median for White men. Further, another study found, over four decades, Black and Latina/o/x students steadily maintained significantly lower high school completion rates than White students (Stark \& Noel, 2015). Despite a common belief that equal opportunities exist for all, many racial disparities persist.

Third, scholars also described how racism could manifest in more covert forms (Sue et al., 2007). Subtle discrimination has been coined in different ways (e.g., aversive racism, modern racism, etc.), the term "microaggression" is often used commonly in academia to describe the subtle, often unconscious, ways that people's biases influence their language and behaviors (Nadal, 2013; Nadal, 2018; Sue, 2010; Torino, Rivera, Capodilupo, Nadal, \& Sue, 2019). The term was first coined by Chester Pierce and his colleagues, defined as "subtle, stunning, often automatic, and non-verbal exchanges which are 'put-downs" (Pierce, Carew, PierceGonzalez, \& Willis, 1978, p. 66). While some articles were written about microaggressions in the three decades following its conceptualization (e.g., Solórzano, 1998), the concept did not gain mainstream popularity until counseling psychologist Derald Wing Sue and colleagues (2007) reintroduced and reconceptualized the term in the American Psychologist. Over the past ten years, hundreds of academic works (Nadal et al., 2016; Wong et al., 2014) and thousands of media articles on microaggressions have emerged (Nadal, 2018); accordingly, MerriamWebster Dictionary added "microaggression" as an entry in 2017 (Italie, 2017).

Previous scholars have hypothesized that the cumulative impact of these three diverse types of racism - overt racism, systemic and structural racism, and racial microaggressions - can result in trauma, otherwise known as racial trauma (Bryant-Davis, 2007; Bryant-Davis \& Ocampo, 2006, Comas-Díaz, 2016). When people of color experience trauma related to race or ethnicity, they are more likely to undergo behavioral or personalityrelated changes that are often pervasive and long-lasting and align with typical symptoms of Post-Traumatic Stress Disorder (PTSD; Carter \& Sant-Barker, 2015). However, because trauma is defined by the Diagnostic Statistical Manual of Mental Disorders - Fifth Edition (DSM-V) (American Psychiatric Association, 2013), as an event where "actual or threatened death, serious injury, or sexual violation" (p. 271) occurs, not all racial traumas would be considered for a PTSD diagnosis. Using rigid definitions of trauma, the only types of racial traumas that would be considered legitimate traumas would be those in which an individual survived through or witnessed death, life-threatening injury, or sexual violations (e.g., experiencing or witnessing a violent hate crime, surviving a racially-motivated sexual assault). In this way, people of color who exhibit trauma symptoms and fit PTSD criteria, yet do not experience trauma as defined by the DSM-V, may not be diagnosed with PTSD - resulting in practitioners providing them with ineffective or inapplicable mental health treatment (Carter, 2007).

When individuals are diagnosed with PTSD, trauma specialists traditionally attribute the negative symptoms that an individual is facing to an external source or force, instead of some weakness or fault of 
the individual. For instance, when war veterans or survivors of sexual assault begin to experience symptoms such as disassociation or avoidance, they are often told by medical and mental health practitioners that their symptoms are "normal" and "expected" responses to the trauma they experienced. However, by not naming racism or other forms of oppression as a legitimate type of trauma, people of color (and others) continue to internalize that they are not coping with discrimination effectively, instead of externalizing the role of historical and systemic oppression in their lives (Comas-Diás, 2016, Nadal, 2018). People who struggle with pervasive and painful experiences with racism are encouraged to reframe their perspectives or to "get over it," instead of being validated that they are experiencing "normal" and "expected" responses to trauma. Further, unlike other more traditionally accepted forms of traumas, people of color who are impaired by racism are unable to entirely remove themselves from the trauma source (i.e., they may encounter racism at work, in public spaces, through racist laws and policies, etc.), increasing the likelihood of being retraumatized continually over time.

The purpose of this paper is to understand the concept of microaggressive trauma, or "the excessive and continuous exposure to subtle discrimination (both interpersonal and systemic) and the subsequent symptoms that develop or persist as a result" (Nadal, 2018, p. 13). Because people of color experience microaggressions regularly in their lives, they may have an array of emotional, cognitive, and psychological reactions that often lead to psychological and physical health consequences (Sue, 2010; Torino et al., 2019). However, when microaggressions are so pervasive (i.e., they are experienced with high intensity), they significantly impair a person's daily functioning, may cause significant psychological distress, and may activate or exacerbate typical PTSD symptoms - including, but not limited to - hypervigilance, anxiety, avoidant behavior, and intrusive thoughts (Nadal, 2018).

\section{Review of Racial Microaggressions}

Sue and colleagues (2007) defined racial microaggressions as "brief and commonplace daily verbal, behavioral, and environmental indignities, whether intentional or unintentional, that communicate hostile, derogatory, or negative racial slights and insults to the target person or group" (p. 271). Microaggression Theory (Nadal, 2013; Torino et al., 2019) expanded this definition to recognize the impact of microaggressions on other historically marginalized groups (e.g., gender, sexual orientation, gender identity, ability, religion, size, age, social class, and others). The use of the word "micro" does not describe the quality of these offenses (which are not small and irrelevant) but symbolizes the covert and individual manner in which this type of discrimination occurs - which often makes it difficult to detect, pinpoint or prove (Sue, 2010).

Previous research supports that microaggressions are predictors of depressive symptoms (e.g., Nadal, Griffin, Wong, Hamit, \& Rasmus, 2014; Ong, Burrow, Fuller-Rowell, Ja, \& Sue, 2013); anxiety and alcohol symptoms (e.g., Blume, Lovato, Thyken, \& Denny, 2012); sleep disturbance (Ong, Cerrada, Lee, \& Williams, 2017); physical health issues (Nadal, Griffin, Wong, Davidoff, \& Davis, 2014; Walls, Gonzalez, Gladney, \& Onello, 2015); and suicidal ideation (O'keefe, Wingate, Cole, Hollingsworth, \& Tucker, 2015). Previous studies have also uncovered ways microaggressions affect people based on their multiple identities (e.g., Balsam, Molina, Beadnell, Simoni, \& Walters, 2011; Lewis \& Neville, 2015; Nadal, Mazzula, Rivera, \& Fujii-Doe, 2014; Nadal, Wong, Sriken, Griffin, \& Fujii-Doe, 2015), suggesting people encounter more microaggressions when they have multiple marginalized identities.

Despite these numerous studies, previous authors have described microaggressions theory as being "pure nonsense" (Thomas, 2008, p. 74) or having "inadequate evidence" to support the existence of microaggressions (Lilienfeld, 2017, p. 138). When people claim microaggressions are merely imagined or that members of other historically marginalized groups are too sensitive or paranoid, they are essentially committing a microaggression themselves. While some may label such behavior as "victim blaming" (Sue, Capodilupo, Nadal, \& Torino, 2008) or "whitesplaining" (Achola, 2015), their assertion that there are always absolute, alternative explanations to 
microaggressions- unrelated to race, ethnicity, or other identities- negates the lived experiences of people who can report multiple experiences with oppression.

Two known empirical studies demonstrate the relationship between racial microaggressions on trauma symptoms. In a study with Latina/o/x participants, Torres and Taknint (2015) found experiences with racial microaggressions predicted the number of trauma symptoms experienced - with ethnic identity and self-efficacy that served as moderators between the two variables. In a study with Black women participants, Moody and Lewis (2019) reported that gendered racial microaggressions predicted trauma symptoms, and that internalized oppression moderated the two variables. While both studies provide initial evidence of the significant connection between racial microaggressions and trauma symptoms for two specific subgroups, it would be essential to understand if, and how, racial microaggressions correlate with trauma symptoms for other subgroups, or people of color in general.

\section{Review of the Current Study}

The current study used a correlational cross-sectional design aimed to examine the relationship between racial microaggressions, racial trauma, and PTSD symptoms. Two main hypotheses include:

H1) participants who report experiencing more racial microaggressions will report more trauma symptoms than those who report experiencing less racial microaggressions.

H2) participants who report a racially- or culturally-related trauma will report more trauma symptoms than those who do not report racially or culturally-related trauma.

Exploratory research questions will examine differences in race, gender, sexual identity, religion, educational level, birthplace, and social class, as well as whether different types of microaggressions are associated with trauma symptoms.

\section{Methods}

\section{Participants}

The sample consisted of 254 participants; $70.9 \%$ identified as female $(n=180), 22.0 \%$ identified as male $(n=56)$, and $7.1 \%$ identified as transgender or gender nonbinary $(n=18)$. The average age of the sample was 34.87 ( $S D=10.65$ years, with an age range of 18 to 69 ). Open-ended participant data was coded into six major racial/ethnic groups: Asian American or Pacific Islander (AAPIs; $n=86$, or 33.9\%), Black $(n=77$, or $30.3 \%$ ), Multiracial $(n=43$, or $16.9 \%)$, Latina/o/x or Hispanic $(n=40$, or $15.7 \%)$, Native American $(n=4$, or $1.6 \%)$, and Middle Eastern $(n=4$, or $1.6 \%)$. A variety of specific ethnic identities were reported, including: 52 Filipino Americans, 49 Black or African Americans, 20 Chinese Americans, 18 South Asian Americans (e.g., Indian, Pakistani), 18 Mexican Americans, 13 West Indian or Caribbean Americans (e.g., Jamaican, Haitian), 11 Puerto Ricans, and 7 Korean Americans. The remaining 66 participants listed multiethnic backgrounds or other unique ethnicities.

When considering sexual orientation, participants identified as heterosexual ( $n=149$, or 58.7\%); lesbian/ gay $(n=34$, or $13.4 \%)$; bisexual, pansexual, or fluid ( $n=33$, or $13 \%)$; queer $(n=24$, or $9.4 \%)$; or asexual $(n=1$, or $0.4 \%)$. Ten participants identified their sexual orientation in unique ways (3.9\%) and the remaining did not report sexual orientation $(n=3$, or $1.2 \%)$. Majority of participants were born in the US $(n=198$, or $78 \%)$ and 56 participants (or 22\%) were born outside of the US. Majority of participants resided in the Northeast $(n=99$, or 39\%); the remainder were from West Coast $(n=60$, or $23.6 \%)$; Southeast/Mid-Atlantic $(n=35$, or $13.8 \%)$; Midwest ( $n=25$, or 9.8\%); Southwest ( $n=15$, or 5.9\%); Alaska/Hawai'i $(n=8$, or $3.1 \%)$; Rocky Mountains $(n=$ 5 , or $2 \%$ ); and 7 participants (or $2.8 \%$ ) lived somewhere else. 
Participants self-identified social class in many ways: middle class $(n=121$, or $47.6 \%)$, lower middle class or working class $(n=42$, or $16.5 \%)$, upper middle class $(n=27$, or $10.6 \%)$, poor $(n=17$, or $6.7 \%)$, and upper class $(n=4$, or $1.6 \%)$. The remaining $16.9 \%$ of participants did not report social class. The sample was highly educated, with majority having Master's degrees $(n=78$, or $30.7 \%)$; Bachelor's degrees $(n=70$, or $27.6 \%)$; or doctorate degrees $(n=47$, or $18.5 \%)$. Some participants reported a high school diploma or less $(n=34$, or $13.4 \%)$ or an Associate's degree $(n=25$, or 9.8\%). Finally, the largest religious or non-religious groups included Christians $(n=60$, or 23.6\%), Catholics $(n=43$, or 16.9\%), Agnostics $(n=19$, or 7.5\%), Spiritual $(n=19$, or $7.5 \%)$, Atheists $(n=16$, or $6.3 \%)$, No Religion $(n=16$, or $6.3 \%)$, Jewish $(n=3$, or $1.2 \%)$, Buddhists $(n=9$, or $3.5 \%)$, Hindu ( $n=5$, or $2.0 \%)$, Muslim $(n=4$, or $1.6 \%)$, Pagan $(n=1$, or $0.4 \%)$, or Sikh $(n=2$, or $0.8 \%)$. Many participants skipped this question about religious/non-religious identity $(n=41$, or $16.1 \%)$, and the remaining 16 participants (or $7.5 \%$ ) self-identified in unique ways.

\section{Recruitment}

After receiving approval from the Institutional Review Board (\#2016-07530), all measures were adapted into an online format and were uploaded to the survey platform, SurveyMonkey.com. The researchers utilized a purposive sampling technique to obtain an ethnically diverse sample. A recruitment advertisement with a survey link was created and distributed to various community organizations listservs (e.g., Asian American Psychological Association and LGBTQ Scholars of Color Network). Various social media platforms were utilized, including public Facebook pages (e.g., The Center for LGBTQ Studies, the Filipino American National Historical Society) and various targeted Facebook groups (e.g., Black Lives Matter chapters, Latinos Unidos, "I love being Black", etc.).

\section{Measures}

Demographic survey. Participants were asked to complete an open-ended demographic questionnaire inquiring about age, education level, gender identity, sexual orientation, religion, socioeconomic status, years in the US, birthplace, and current geographic location. The practice of open-ended demographic forms has been suggested as standardized practice, as forced choices are often to considered to exclusionary toward multiracial people (Townsend, Markus, \& Bergsieker, 2009), transgender and gender-nonconforming people (Smiler, 2017), and any group with identities that are nonbinary or are historically excluded on standardized forms.

Racial and Ethnic Microaggression Scale (REMS). Participants were asked to complete the Racial and Ethnic Microaggressions Scale-45 (REMS-45; Nadal, 2011) to assess the frequency of experienced microaggressions based on race and ethnicity. The REMS-45 is composed of 45 items and asked the respondent to identify whether or not they experienced the microaggression in the past six months. Participants scored " 0 " for "I did not experience this event in the past six months" or " 1 " = "I experienced this event at least once in the past six months." The 45 items are loaded onto six factors: Subscale 1: Assumptions of Inferiority, Subscale 2: Second Class Citizens and Assumptions of Criminality, Subscale 3: Microinvalidations, Subscale 4: Exoticization/ Assumptions of Similarity, Subscale 5: Environmental Microaggressions, and Subscale 6: Workplace and School Microaggressions. For the current study, the REMS-45 yielded a Cronbach's alpha of .90, with subscale alphas ranging from .70 to .88 .

PTSD Checklist-5. The PTSD Checklist (PCL-5; Blevins et al., 2015) measures PTSD symptoms using DSM-5 criteria, consisting of two parts. First, respondents were asked to identify the worst event or events that have ever occurred if they felt comfortable doing so. They were then asked about how long ago it happened; if it involved an actual or threatened death, serious injury, or sexual violence, how they experienced the event, and whether or not the event was accidental or caused by a natural disaster. The second part of the PCL-5 is a checklist of symptoms and the magnitude to which the respondent has been bothered by a symptom within the past month. Individuals responded on a five-point scale $(0=$ not at all, $1=$ a little bit, $2=$ moderately, $3=$ quite a bit, 
$4=$ extremely), with total scores ranging from 0 to 80 . For the current study, the PCL-5 yielded a Cronbach's alpha of .96.

Racially- or Culturally-Related Trauma. After identifying "worst event or events", participants were asked if the trauma they identified was related to their "race, ethnicity, or other cultural identities" and were presented with three choices: "yes," "no," or "not sure." Participants were then invited to elaborate qualitatively if they felt comfortable.

\section{Data Cleaning and Coding}

Because we used an online sample, data were examined both statistically and graphically for outliers. First, with an initial pool of 594 participants, we removed White participants $(n=197)$, and participants did not complete all of the measures $(n=141)$. Second, raw data were also evaluated to determine if any participants were fraudulent or if participants completed the survey more than once. Third, to assess missing data, we used Little's (1987) Missing Completely at Random (MCAR) tests; results indicated REMS, $\chi 2$ (997) 1083.81, $p=.028$ and PCL-5, $\chi 2$ (189) 152.52, $p=.976$, supporting that data were missing completely at random. Finally, because open-ended measures were used, the research team coded participants' self-reported identities into categorical variables, using a coding scheme. These codes were formed based on most common demographic groups (e.g., race was recoded into Black, AAPI, Latina/o/a, Middle Eastern, Native American, and Multiracial); while also including non-binary conceptualizations (e.g., gender was categorized as female, male, and transgender/ gender-nonconforming).

\section{Procedure}

Data were collected online from April 2017 until April 2018. The average time to complete the entire study was 9 minutes. Participants were not compensated in any way. A coding scheme was created for qualitative data acquired from the demographic questionnaire. Data were entered and analyzed in IBM (2017) SPSS statistics software. Correlations and hierarchical regressions were conducted to interpret the relationships between independent and dependent variables.

\section{Results}

\section{Preliminary Analyses}

Descriptive data regarding the prevalence of racial microaggression experiences and trauma symptoms were examined. Means and standard deviations for the total population, and various groups are shown in Table 1. Total PCL scores ranged from 0 to 80 , with an average score of $21.53(S D=19.43)$. Sixtysix participants scored higher than 33, which is traditionally the lowest score needed for a PTSD diagnosis (Blevins et al., 2015). Regarding the type of trauma, over half ( $n=140$, or $55.1 \%$ ) identified an actual or threatened death, serious injury, or sexual violence (i.e., DSM criteria for trauma), while the other half ( $n=$ 87 or $34.3 \%$ ) reported the event did not involve these instances and 27 participants did not know. Sixty-two percent of participants $(n=157)$ experienced the trauma directly; 33 participants (or 13\%) learned about it from a close family member or friend, and 20 participants (or 7.9\%) witnessed it. The remaining were repeatedly exposed to the trauma through their jobs $(n=1$, or $0.4 \%)$ or described some other circumstance $(n=15$, or $5.9 \%)$. Majority of participants $(n=186$, or $73.2 \%)$ said the event did not involve death of a close family member or friend, while some say it involved an accident or violence towards a family member or friend ( $n=23$, or $9.1 \%$ ) and others say it involved natural causes $(n=17$, or $7.14 \%)$. Regarding racial trauma, 97 participants reported that race or culture was involved in their traumatic experience, 82 participants reported that race or culture was not involved in their trauma experience, and 39 participants were not sure. 
Table 1. Means and Standard Deviations for REMS and PCL Scores for Gender, Race, Sexual Orientation, Social Class, and Educational Background

\begin{tabular}{|c|c|c|c|c|c|}
\hline & & & & PCL & \\
\hline & $\mathbf{N}$ & Mean & SD & Mean & SD \\
\hline Gender & & & & & \\
\hline Female & 180 & 20.57 & 8.75 & 20.64 & 17.93 \\
\hline Male & 56 & 22.02 & 10.46 & 19.80 & 20.01 \\
\hline $\begin{array}{l}\text { Transgender or Gender } \\
\text { Nonconforming }\end{array}$ & 18 & 25.50 & 9.18 & 35.89 & 26.53 \\
\hline Race & & & & & \\
\hline Black & 77 & 21.05 & 9.10 & 23.61 & 20.10 \\
\hline Asian American & 86 & 20.30 & 8.28 & 17.47 & 16.15 \\
\hline Latina/o/x & 40 & 25.92 & 11.14 & 26.95 & 21.32 \\
\hline Multiracial & 43 & 20.26 & 7.61 & 24.16 & 21.34 \\
\hline Indigenous & 4 & 11.67 & 9.87 & 1.00 & 1.41 \\
\hline Arab/Middle Eastern & 4 & 16.25 & 12.42 & 7.25 & 5.25 \\
\hline Sexual Orientation & & & & & \\
\hline Heterosexual & 149 & 20.33 & 9.59 & 18.24 & 16.33 \\
\hline Lesbian or Gay & 34 & 19.21 & 8.50 & 21.00 & 19.87 \\
\hline Bisexual or Pansexual & 33 & 25.10 & 6.78 & 32.24 & 22.37 \\
\hline Queer & 23 & 24.65 & 8.21 & 25.54 & 21.20 \\
\hline Social Class & & & & & \\
\hline Poor/Poverty/Lower & 17 & 25.56 & 9.91 & 35.82 & 22.32 \\
\hline Working/Lower Middle & 42 & 22.58 & 9.09 & 26.02 & 22.42 \\
\hline Middle & 121 & 21.25 & 9.57 & 19.78 & 17.98 \\
\hline Upper Middle & 27 & 19.50 & 8.04 & 11.22 & 11.94 \\
\hline Upper/ Wealthy & 4 & 17.75 & 13.50 & 7.50 & 5.26 \\
\hline Highest Level of Education & & & & & \\
\hline High School & 34 & 19.40 & 9.21 & 23.62 & 19.35 \\
\hline Associates & 25 & 21.77 & 10.08 & 28.60 & 20.19 \\
\hline Bachelors & 70 & 22.70 & 9.08 & 26.11 & 20.27 \\
\hline Masters & 78 & 19.66 & 8.94 & 18.51 & 18.80 \\
\hline Doctorate & 47 & 22.76 & 9.32 & 14.47 & 16.10 \\
\hline
\end{tabular}




\section{Main Analyses}

To first explore the relationship between racial microaggressions and trauma, correlations were run between REMS-Total score, REMS-Subscale scores, and PCL scores. Results indicate a significant correlation between REMS-Total and PCL scores $(r=.417, N=226 . p<.001$, two-tailed). Five of the six REMS subscales (all except Subscale 5: Environmental Microaggressions) were positively and significantly correlated with PCL average scores, with $r$-scores ranging between .25 to .42 ( $p<.001$, two-tailed). All correlations are presented in Table 2.

Table 2. Correlations between PCL, REMS, and REMS-Subscales

\begin{tabular}{|c|c|c|c|c|c|c|c|c|}
\hline & PCL & $\begin{array}{c}\text { REMS- } \\
\text { Total }\end{array}$ & REMS1 & REMS2 & REMS3 & REMS4 & REMS5 & REMS6 \\
\hline PCL & 1 & & & & & & & \\
\hline REMS-Total & $.42^{* *}$ & 1 & & & & & & \\
\hline REMS1 & $.32^{* *}$ & $.72^{* *}$ & 1 & & & & & \\
\hline REMS2 & $.29^{* *}$ & $.60^{* *}$ & $.62^{* *}$ & 1 & & & & \\
\hline REMS3 & $.27^{* *}$ & $.74^{* *}$ & $.41^{* *}$ & $.28^{* *}$ & 1 & & & \\
\hline REMS4 & $.25^{* *}$ & $.66^{* *}$ & $.22^{* *}$ & $.19^{* *}$ & $.38^{* *}$ & 1 & & \\
\hline REMS5 & .03 & $.26^{* *}$ & $-1.47^{*}$ & $-.19^{* *}$ & $.14^{* *}$ & $.23^{* *}$ & 1 & \\
\hline REMS6 & $.36^{* *}$ & $.74^{* *}$ & $.56^{* *}$ & $.41^{* *}$ & $.46^{* *}$ & $.42^{* *}$ & .06 & 1 \\
\hline
\end{tabular}

Note: PCL = PTSD Checklist; REMS = Racial and Ethnic Microaggressions Scale; REMS1 = Assumptions of Inferiority; REMS2 = Second-Class Citizen and Assumption of Criminality; REMS3 = Microinvalidations; REMS4 = Exoticization and Assumptions of Similarity; REMS5 = Environmental Microaggressions; REMS6 = School and Workplace Microaggressions.

** Correlation is significant at the 0.01 level (2-tailed).

${ }^{*}$ Correlation is significant at the 0.05 level (2-tailed).

To test Hypothesis 1, we conducted a hierarchical regression analysis with control variables (age, race, sexual orientation, religion, socioeconomic status, geographic region, educational background, and birthplace) in Step 1, REMS in Step 2, and the presence of racially- or culturally-related trauma in Step 3. As noted in Table 3 , the overall regression model in Step 1 was significant, $F(9,156)=3.64, p<.001$, accounting for $12.6 \%$ of the variance $\left(R^{2}=.126\right)$. Three variables yielded a significant association with trauma: sexual orientation, $\beta=.27$, $t(156)=3.58, p=.001 ;$ social class, $\beta=-.21, t(156)=-2.84, p=.001$; and educational background, $\beta=-.18, t(156)=$ $-2.44, p=.02$. In Step 2 , the overall regression model was also significant, $F(10,155)=7.07, p<.001$, accounting for $26.9 \%$ of the variance $\left(R^{2}=.126\right)$. Again, sexual orientation, $\beta=.24, t(155)=3.46, p=.001$, social class, $\beta=-.14$, $t(155)=-2.05, p=.04$, and educational background, $\beta=-.21, t(155)=-3.07, p=.001$, were significantly associated with greater trauma symptoms. Supporting Hypothesis 1, REMS was also found to be significantly associated with trauma, $\beta=.39, t(155)=5.61, p=.001$. In Step 3, the overall regression model was significant, $F(11,154)$ $=6.47, p<.001$, accounting for $26.7 \%$ of the variance. Sexual orientation, educational background, and REMS scores were significantly associated with trauma. However, in contrast to our prediction in Hypothesis 2, the presence of a racially- or culturally-related trauma was not associated with trauma symptoms, $\beta=-.06, t(154)=$ $7.94, p=.43$.

To further test Hypothesis 2 (whether the presence of a racially- or culturally-related trauma influenced trauma symptoms), we conducted a One-way Analysis of Variance (ANOVA). Results indicated the groups were significantly different, $F(1,225)=5.87, p=.02$. Participants who reported that race or culture was involved 
in their trauma experience scored highest PCL scores $(x=24.98, S D=22.29)$, followed by participants who were unsure if race or culture were involved $(x=21.97, S D=18.17)$, and participants who reported that race or culture was not involved in their trauma experience $(x=15.60, S D=13.80)$.

Table 3. Hierarchical Regression Analysis Predicting Traumatic Stress Symptoms from Racial Ethnic Microaggressions

\section{Coefficient}

B

Step 1

Gender

Age

Race

Sexual Orientation

Religion

Social Class

Geographic Region

Highest Degree

Birthplace

Step 2

Gender

Age

Race

Sexual Orientation

Religion

Social Class

Geographic Region

Highest Degree

Birthplace

REMS

Step 3

Gender

Age

Race

Sexual Orientation

Religion

Social Class

Geographic Region

Highest Degree

Birthplace

REMS

Race Involved
Std. Error

$\begin{array}{ll}1.06 & 2.47 \\ 0.16 & 0.14 \\ -1.88 & 1.22 \\ 4.62 & 1.29 \\ 0.39 & 0.41 \\ -3.49 & 1.23 \\ -0.39 & 0.75 \\ -2.90 & 1.19 \\ 0.54 & 3.53\end{array}$

$-0.20$

0.17

$-1.37$

4.10

0.35

$-2.34$

$-0.64$

$-3.35$

0.86

0.81

$-0.15$

0.18

$-1.29$

4.16

0.33

$-2.16$

$-0.65$

$-3.33$

0.77

0.80

1.57

2.27

1.12

1.18

0.38

1.14

0.69

1.09

3.23

0.15
Collinearity Statistics

95.0\% CI Tolerance

VIF

$\begin{array}{ccccc}0.43 & -3.82 & 5.94 & 0.92 & 1.09 \\ 1.19 & -0.11 & 0.43 & 0.95 & 1.05 \\ -1.54 & -4.29 & 0.54 & 0.93 & 1.07 \\ 3.58^{\star * *} & 2.07 & 7.17 & 0.91 & 1.10 \\ 0.94 & -0.43 & 1.20 & 0.90 & 1.12 \\ -2.84^{\star *} & -5.92 & -1.07 & 0.94 & 1.07 \\ -0.52 & -1.87 & 1.09 & 0.98 & 1.02 \\ -2.45^{\star} & -5.24 & -0.56 & 0.95 & 1.05 \\ 0.15 & -6.44 & 7.52 & 0.95 & 1.06\end{array}$

$-0.0$

1.35

$-1.23$

$3.46 * * *$

0.93

$-2.05 *$

$-0.93$

-3.08 **

$\begin{array}{ll}-4.69 & 4.28\end{array}$

0.91

1.10

0.27

$\begin{array}{ll}-0.08 & 0.41\end{array}$

0.95

1.05

$-3.59 \quad 0.84$

0.93

1.08

$1.76 \quad 6.44$

0.90

1.11

$-0.40 \quad 1.09$

0.90

1.12

$-4.60-0.09$

0.91

1.11

$\begin{array}{ll}-1.99 & 0.72\end{array}$

0.98

1.02

$-5.49-1.20$

0.94

1.06

$5.61^{* * *}$

$-5.53 \quad 7.24$

0.95

1.06

$0.53 \quad 1.10$

0.93

1.08

Note. $\mathrm{REMS}=$ Racial and Ethnic Microaggressions Scale; $\mathrm{CI}=$ confidence interval; $\mathrm{VIF}=$ variance inflation factor; ${ }^{\star} \mathrm{p}<.05 .{ }^{\star *} \mathrm{p}<.01$. ${ }^{\star * *} \mathrm{p}<.001$. 
To answer our exploratory research questions, we first conducted a multivariate analysis of variance (MANOVA) to determine if race/ethnicity, gender, sexual orientation, socioeconomic status, and educational status correlated with racial microaggressions (i.e., REMS scores) or trauma (i.e., PCL scores). Results indicate that there were significant differences between racial groups in both REMS scores, $F(5,220)=3.18, p=.01$ and PCL scores, $F(5,248)=3.16, p=.01$, with Latina/o/x participants reporting highest mean scores on both scales. There were significant differences in PCL scores based on gender, $F(2,251)=5.51, p=.001$, and sexual orientation, $F(3$, $247)=4.45$, $p=.001$, with transgender and gender nonconforming (TGNC) participants reporting significantly higher PCL scores than both cisgender women and cisgender men, and that all non-heterosexual participants reported higher PCL scores than heterosexual participants. ANOVAs revealed significant differences in PCL scores based on social class $F(5,230)=4.94, p=.001$, and educational level, $F(4,249)=4.11$, $p=.001$, with highest PCL scores reported by people who identified as poor, living in poverty, or lower class, as well as people with Associate's degrees. All mean scores are reported in Table 1.

Finally, to explore whether specific types of microaggressions predicted trauma symptoms, hierarchical regression was utilized, with Step 1, including all control as mentioned above variables and Step 2, examining all six REMS subscales. Again, a significant overall regression model emerged, $F(15,165)=4.38, p<.001$, with Subscale 6: School and Workplace Microaggressions, $\beta=.17, t(219)=2.12, p=.04$, emerging as the only significant predictor of trauma symptoms.

\section{Discussion}

The current study aimed to broaden our understanding of the detrimental effects of racial microaggressions by examining its relationship to racial trauma and trauma symptoms. Results indicated that a higher amount of racial microaggressions was associated with a higher number of traumatic symptoms. The significant $R^{2}$ change statistics between Step 1 and Step 2 (approximately 14.3\%) suggests that racial microaggressions predict variance in trauma symptoms, above and beyond that predicted by the control variables. So, while racial microaggressions have been found that predict other mental health variables like deression, anxiety, and low selfesteem (see Torino et al., 2019 for a review) and that racial discrimination, in general, predicts trauma symptoms (Chou et al., 2012), the current results suggest a significant correlation between racial microaggressions and symptoms of psychological trauma. It should also be noted that the current study produced larger effect sizes than previous studies that reported racial microaggressions to significantly predict depression. For example, Nadal and colleagues (2014) found racial microaggressions as significant predictors of depression and other mental health variables, only 1.4 to $2.6 \%$ of the variance was accounted. Thus, these results suggest that racial microaggressions may be more significant predictors of traumatic symptoms than microaggressions predict depression symptoms.

While the ANOVA supported the hypothesis that participants who report a trauma involving or race or culture are more likely to report higher PCL scores, our hierarchical regression did not support this hypothesis. Thus, future research may further examine how the presence of race and culture during a traumatic event may influence the quantity or quality of trauma symptoms. Future research may also examine if microaggressions may mediate the relationship between racial trauma and trauma symptoms, mainly when the trauma people experience involves something related to racism or discrimination. Such findings may be related to Nadal's (2018) hypothesis that microaggressions can be re-traumatizing of individuals' past experiences of discrimination, and how triggers of past traumas may activate or intensify certain memories. Furthermore, microaggressions can be considered a form of complex trauma, or "a type of trauma that occurs repeatedly and cumulatively, usually over some time and within specific relationships and contexts" (Courtois, 2008, p. 86). Complex trauma can involve various traumatic events that are related (e.g., repeated racial microaggressions by a specific perpetrator in a 
workplace or school environment) or unrelated (e.g., a collection of microaggressions by various perpetrators in varied environments and periods of an individual's life).

Results indicated that sexual orientation was associated with trauma symptoms at all steps in the regression analyses; transgender or gender non-conforming people report higher levels of trauma symptoms than both cisgender men or women; and queer people report more trauma symptoms than heterosexual people. These findings suggest lesbian, gay, bisexual, transgender, and queer (LGBTQ) people of color may experience more trauma symptoms than their heterosexual/cisgender counterparts, and that their trauma symptoms increase when they experience racial microaggressions. Given that LGBTQ people of color report an array of traumatic life events, due to the intersection of their race, ethnicity, gender identity, and other factors (Singh \& McKleroy, 2011), more quantitative research is needed to understand the negative impact of trauma and wellbeing of LGBTQ people of color. Further, considering the disproportionate amount of hate violence faced by LGBTQ people of color - especially transgender women of color (Nadal, 2018), it is important for psychologists and other practitioners to vigilantly recognizing the salience of intersectional identities on trauma and other mental health factors.

Finally, social class and educational status were found to be significantly associated with trauma symptoms at multiple steps of the model - with people of lower social classes and those who are less educated reporting more trauma symptoms. Given the results which indicated that racial microaggressions that occur in the workplace or school settings are significantly associated with trauma, it is critical to understand how microaggression that occurs in settings of places of learning or employment can be detrimental and potentially traumatizing. Taken together, such findings highlight the need to advocate for people of historically marginalized or disenfranchised people, particularly when they have less access to financial or educational resources.

\section{Limitations}

Several limitations of the current study should be noted. First, the correlational and cross-sectional nature of the methodology restricted us from drawing any causal conclusion regarding the effects of racial microaggression experiences on trauma symptoms. Second, the use of a self-report online survey makes the study vulnerable to recall bias or memory errors, careless mistakes. Additionally, the order in which the measures were presented could have led to a priming effect. Third, the REMS measures microaggressions that occurs in 6 months, while the PCL-5 asks for trauma symptoms in the last month; hence, it is unclear if any trauma symptoms preceded experiences of racial microaggressions. Relatedly, both the REMS and the PCL measure the presence of the variable, but not the impact of the severity of the trauma symptoms. Further, while the sample was racially diverse, we did not examine ethnic differences; disaggregating this data would be imperative for understanding within-group differences, particularly for racial groups in which vast disparities exist. For example, for Asian Americans and Latina/o/x Americans, issues like ethnicity, skin color, phenotype, social class, religion, and colonial mentality have influenced how individuals uniquely experience microaggressions (Nadal, Mazzula, Rivera, \& Fujii-Doe, 2014; Nadal et al., 2015).

\section{Implications for Counseling and Psychology}

Counselors, psychologists, and other helping professionals can integrate the study's findings into their professional work in a variety of ways. First, results provide more empirical support for recognizing how racial microaggressions negatively impact the health and wellness of communities of color. Helping professionals may consider how microaggressions manifest on all levels in their workplaces - interpersonally (e.g., between colleagues, employees, supervisors, and clients); on group-levels (e.g., through racialized group or power dynamics); and institutionally (e.g., through biased policies) and advocate for social justice on multiple levels.

Future researchers can further examine racial trauma and microaggressive trauma as concepts that negatively impact the lives of people of color and prohibit their ability to thrive in all aspects of their lives. Researchers can examine if microaggressions can perhaps trigger past experiences of racial trauma similar 
to retraumatizations. Scholars have found retraumatization to be detrimental to psychological health (see Duckworth \& Follette, 2012), while others have supported that accumulative or complex trauma and revictimization often results in greater symptomology (Courtois, 2008). So, while racial microaggressions may appear innocuous or harmless, they may trigger memories of intensity or frequent racial discrimination, which may exacerbate trauma symptoms. Intersectional research would be especially important, particularly for members of multiple marginalized groups; for instance, microaggressive trauma can be further understood by replicating this study with microaggressions measures developed for other groups (e.g., LGBTQ People of Color Microaggressions Scale; Balsam et al., 2011). In doing so, research can be used to inform policy and practice in counseling psychology and beyond.

Specific to clinical practice, helping professionals can consider how microaggressive trauma may impact their clients and conceptualize their symptoms as resulting from systemic oppression, instead of from any individual fault or weakness. Such practices are important given the previous studies that find that even when people of color experience trauma symptoms, they are less likely than Whites to seek treatment (Roberts, Gilman, Breslau, Breslau, \& Koenen, 2011). If people of color (and other historically marginalized groups) felt less blamed for reacting negatively to racial trauma, and instead were categorized with other trauma survivors who are often taught their reactions are "natural" and "expected" responses to trauma (Nadal, 2018, p. 12), perhaps they have a greater ability to thrive in American society. For instance, Comas-Díaz (2016) conceptualized a race-informed model of working with survivors of racial trauma - integrating methods used in trauma-focused research, while including additional steps that account for the effects of systemic racism and historical trauma. In considering race, ethnicity, and other salient cultural identities this trauma-focused therapy, clinicians give clients the opportunity to feel validated and normalized, instead of feeling any potential shame or blame.

Educators can teach their students and trainees about critical approaches to mainstream psychological concepts like trauma; they can also teach their students how to advocate for updated, expanded definitions of such concepts for historically marginalized communities. Finally, as social justice activism has been argued to be an ethical obligation for psychologists and other helping professionals (Nadal, 2017), teaching students about racial trauma, microaggressions, and their influences on mental health are necessary for their academic training, but also for their potential to develop cultural humility and cultural competence in working with future clients. Educating future counselors and practitioners to advocate for new conceptualizations of trauma can help people of historically marginalized groups to thrive.

\section{Corresponding Author}

For correspondence regarding this article, please contact Dr. Kevin Nadal, knadal@jjay.cuny.edu, 524 W. $59^{\text {th }}$ Street, New York, NY 10031, (212) 237-8795 


\section{References}

Achola, E. (2015, September). Whitesplaining-it's not just Matt Damon: Why I set up a black women's blog. The Telegraph. Retrieved from https:/www.telegraph.co.uk/women/womens-life/11890566/ Whitesplaining-not-just-Matt-Damon-Why-I-set-up-a-black-womens-blog.html

American Psychiatric Association ACA. (2013) Diagnostic and statistical manual of mental disorders, (5th ed.). Washington, DC: Author.

American Psychological Association (2010). Amendments to the 2002 'Ethical principles of psychologists and code of conduct. American Psychologist, 65, 493.

Balsam, K. F., Molina, Y., Beadnell, B., Simoni, J., \& Walters, K. (2011). Measuring multiple minority stress: The LGBTQ People of Color Microaggressions Scale. Cultural Diversity and Ethnic Minority Psychology, 17, 163-174. doi:10.1037/a0023244

Blevins, C. A., Weathers, F. W., Davis, M. T., Witte, T. K., \& Domino, J. L. (2015). The posttraumatic stress disorder checklist for DSM-5 (PCL-5): Development and initial psychometric evaluation. Journal of Traumatic Stress, 28, 489-498.

Blume, A. W., Lovato, L. V., Thyken, B. N., \& Denny, N. (2012). The relationship of microaggressions with alcohol use and anxiety among ethnic minority college students in a historically White institution. Cultural Diversity and Ethnic Minority Psychology, 18, 45-54.

Bryant-Davis, T. (2007). Healing requires recognition: The case for race-based traumatic stress. The Counseling Psychologist, 35, 135-143.

Bryant-Davis, T., \& Ocampo, C. (2005). The trauma of racism: Implications for counseling, research, and education. The Counseling Psychologist, 33, 574-578.

Bureau of Labor Statistics (2019). Usual weekly earnings of wage and salary workers: First quarter. Washington DC: Author. Retrieved from https://www.bls.gov/news.release/pdf/wkyeng.pdf

Carter, R. T. (2007). Racism and psychological and emotional injury: Recognizing and assessing race-based traumatic stress. The Counseling Psychologist, 35, 13-105.

Carter, R. T., \& Sant-Barket, S. M. (2015). Assessment of the impact of racial discrimination and racism: How to use the Race-Based Traumatic Stress Symptom Scale in practice. Traumatology, 21, 32-39. http:// dx.doi.org/10.1037/trm0000018

Chou, T., Asnaani, A., \& Hofmann, S. G. (2012). Perception of racial discrimination and psychopathology across three u.s. ethnic minority groups. Cultural Diversity \& Ethnic Minority Psychology, 18, 74-81. http://dx.doi.org/10.1037/a0025432

Comas-Díaz, L. (2016). Racial trauma recovery: A race-informed therapeutic approach to racial wounds. In Alvarez, A.N. (Ed); Liang, C. T. H. (Ed); Neville, H. A. (Eds.). The cost of racism for people of color: Contextualizing experiences of discrimination (pp. 249-272). Washington, DC: American Psychological Association.

Courtois, C. A. (2008). Complex trauma, complex reactions: Assessment and treatment. Psychological Trauma: Theory, Research, Practice, and Policy, S, 86-100.

Desmond, M., Papachristos, A. V., \& Kirk, D. S. (2016). Police violence and citizen crime reporting in the black community. American Sociological Review, 81, 857-876.

Duckworth, M. P., \& Follette, V. M. (Eds.). (2012). Retraumatization: Assessment, treatment, and prevention. New York: Routledge.

Gee, G. C., Spencer, M., Chen, J., Yip, T., \& Takeuchi, D.T. (2007). The association between self-reported racial 
discrimination and 12-month DSM-IV mental disorders among Asian Americans nationwide. Social Science \& Medicine, 64, 1984-1996.

IBM Corp. (2017). IBM SPSS statistics for Macintosh. Version 25.0. Armonk, NY: Author.

Italie, L (2017, February). Ghosting, shade, microaggression hit Merriam-Webster website. Washington Times. Retrieved from: http://www.washingtontimes.com/news/2017/feb/7/ghosting-shade-microaggressionhit-merriam-webster/

Jee-Lyn García, J., \& Sharif, M. Z. (2015). Black lives matter: A Commentary on racism and public health. American Journal of Public Health, 105, e27-e30.

Lewis, J. A., \& Neville, H. A. (2015). Construction and initial validation of the Gendered Racial Microaggressions Scale for Black women. Journal of Counseling Psychology, 62, 289-302.

Lilienfeld, S. O. (2017). Microaggressions: Strong claims, inadequate evidence. Perspectives on Psychological Science, 12, 138-169.

Little, R. J. A. (1988). A test of missing completely at random for multivariate data with missing values. Journal of the American Statistical Association, 83, 1198-1202.

Moody, A. T., \& Lewis, J. A. (2019). Gendered Racial Microaggressions and Traumatic Stress Symptoms Among Black Women. Psychology of Women Quarterly, 43, 201-214.

Nadal, K. L. (2011). The Racial and Ethnic Microaggressions Scale (REMS): Construction, reliability, and validity. Journal of Counseling Psychology, 58, 470-480.

Nadal, K. L. (2018). Microaggressions and traumatic stress. Washington DC: American Psychological Association.

Nadal, K. L. (2016). Addressing Trauma Among Gay, Bisexual, and Queer Boys of Color (Issue Brief). Princeton, NJ: Robert Wood Johnson Foundation.

Nadal, K. L. (2017). "Let's get in formation”: On Becoming a Psychologist-Activist in the 21st Century. American Psychologist.

Nadal, K. L. (2018). Microaggressions and Traumatic Stress: Theory, Research, and Clinical Treatment. Washington DC: American Psychological Association.

Nadal, K. L., Griffin, K. E., Wong, Y., Hamit, S., \& Rasmus, M. (2014). Racial microaggressions and mental health: Counseling clients of color. Journal of Counseling and Development. 92, 57-66.

Nadal, K. L., Griffin, K. E., Wong, Y., Davidoff, K. C., \& Davis, L. S. (2017). The injurious relationship between racial microaggressions and physical health: implications for social work. Journal of Ethnic \& Cultural Diversity in Social Work, 26, 6-17.

Nadal, K. L., Mazzula, S. L., Rivera, D. P., \& Fuji-Doe, W. (2014). Microaggressions and Latina/o Americans: An Analysis of Nativity, Gender, and Ethnicity. Journal of Latina/o Psychology, 2, 67-78.

Nadal, K. L., Whitman, C. N., Davis, L. S., Erazo, T., \& Davidoff, K. C. (2016). Microaggressions toward lesbian, gay, bisexual, transgender, queer and genderqueer people: A review of the literature. Journal of Sex Research, 53, 488-508.

Nadal, K. L., Wong, Y., Sriken, J., Griffin, K., \& Fujii-Doe, W. (2015). Racial microaggressions and Asian Americans: An Exploratory study on within-group differences and mental health. Asian American Journal of Psychology, 6, 136-144.

O’keefe, V. M., Wingate, L. R., Cole, A. B., Hollingsworth, D. W., \& Tucker, R. P. (2015). Seemingly harmless 
racial communications are not so harmless: Racial microaggressions lead to suicidal ideation by way of depression symptoms. Suicide and life-threatening behavior, 45, 567-576.

Ong, A. D., Burrow, A. L., Fuller-Rowell, T. E., Ja, N. M., \& Sue, D. W. (2013). Racial microaggressions and daily well-being among Asian Americans. Journal of Counseling Psychology, 60, 188-199. http://dx.doi. org/10.1037/a0031736

Ong, A. D., Cerrada, C., Lee, R. A., \& Williams, D. R. (2017). Stigma consciousness, racial microaggressions, and sleep disturbance among Asian Americans. Asian American Journal of Psychology, 8, 72.

Pierce, C., Carew, J., Pierce-Gonzalez, D., \& Willis, D. (1978). An experiment in racism: TV commercials. In C. Pierce (Ed.), Television and education (pp. 62-88). Beverly Hills, CA: Sage.

Roberts, A. L., Gilman, S. E., Breslau, J., Breslau, N., \& Koenen, K. C. (2011). Race/ethnic differences in exposure to traumatic events, development of post-traumatic stress disorder, and treatment-seeking for post-traumatic stress disorder in the United States. Psychological medicine, 41, 71-83.

Singh, A. A., \& McKleroy, V. S. (2011). "Just getting out of bed is a revolutionary act": The Resilience of transgender people of color who have survived traumatic life events. Traumatology, 17, 34-44.

Smiler, A. P. (2017). Measuring gender. In K. L. Nadal (Ed.) The Sage Encyclopedia of Psychology and Gender. Thousand Oaks, CA: Sage.

Solórzano, D. G. (1998). Critical race theory, race and gender microaggressions, and the experience of Chicana and Chicano scholars. International journal of qualitative studies in education, 11, 121-136.

Stark, P., \& Noel, A. M. (2015). Trends in High School Dropout and Completion Rates in the United States: $1972-$ 2012. Compendium Report. NCES 2015-015. Washington DC: National Center for Education Statistics. Retrieved from https://nces.ed.gov/pubs2015/2015015.pdf

Sue, D. W. (2010). Microaggressions in everyday life: Race, gender, and sexual orientation. New York: Wiley \& Sons.

Sue, D. W., Capodilupo, C. M., Torino, G. C., Bucceri, J. M., Holder, A. M., Nadal, K. L., \& Esquilin, M. E. (2007). Racial microaggressions in everyday life: Implications for counseling. American Psychologist, 62, 271-286.

Sue, D. W., Capodilupo, C. M., Nadal, K. L., \& Torino, G. C. (2008). Racial microaggressions and the power to define reality. American Psychologist, 63, 277-279.

Thomas, K. R. (2008). Macrononsense in multiculturalism. American Psychologist, 63, 74-75.

Torino, G. C., Rivera, D. P., Capodilupo, C. M., Nadal, K. L., \& Sue, D. W. (Eds.) (2019). Microaggression theory: Influence and implications. New York: John Wiley \& Sons.

Torres, L., \& Taknint, J. T. (2015). Ethnic microaggressions, traumatic stress symptoms, and Latino depression: A moderated mediational model. Journal of Counseling Psychology, 62, 393-401. http://dx.doi. org/10.1037/cou0000077

Townsend, S. S., Markus, H. R., \& Bergsieker, H. B. (2009). My choice, your categories: The denial of multiracial identities. Journal of Social Issues, 65, 185-204.

Walls, M. L., Gonzalez, J., Gladney, T., \& Onello, E. (2015). Unconscious biases: racial microaggressions in American Indian health care. The Journal of the American Board of Family Medicine, 28, 231-239.

Wong, G., Derthick, A. O., David, E. J. R., Saw, A., \& Okazaki, S. (2014). The what, the why,and the how: A review of racial microaggressions research in psychology. Race and social problems, 6, 181-200. 